\title{
The Irreducibility of the Bernoulli Polynomial $B_{14}(x)$
}

\author{
By L. Carlitz
}

1. Put

$$
B_{n}(x)=(B+x)^{n}=\sum_{r=0}^{n}\left(\begin{array}{l}
n \\
r
\end{array}\right) B_{r} x^{n-r},
$$

where $B_{r}$ is defined by means of

$$
B_{0}=1, \quad(B+1)^{n}=B^{n} \quad(n>1) .
$$

For $n$ odd, $n>1$, it is familiar that $B_{n}(x)$ has the linear factors $x, x-\frac{1}{2}, x-1$. For $n$ even no factors with rational coefficients are known and it seems plausible that $B_{n}(x)$ is irreducible (with respect to the rational field). The writer has proved [1] that $B_{n}(x)$ is irreducible for

$$
n=k p^{r}(p-1) \quad(1 \leqq k<p, r \geqq 0),
$$

where $p$ is an odd prime, and also for $n=2^{r}, r \geqq 1$. McCarthy [2] has proved the irreducibility of $B_{n}(x)$ for $n=(k p+k+1)(p-1)$ when $k<p$.

It is noted in [1] that for even values of $n \leqq 50$ the irreducibility of $B_{n}(x)$ remains in doubt for $n=14,26,34,37$. The irreducibility of $B_{14}(x)$ has been verified in the Duke University Computing Laboratory by R. Carlitz.

The purpose of the present note is to give a proof of the irreducibility of $B_{14}(x)$ that uses a minimum of computation. While the result is special, the method is of a rather general nature and may perhaps be of use in proving more comprehensive results.

2. Using the notation of Nörlund [2, Ch. 2] we put

$$
P(x)=2^{14} B_{14}\left(\frac{x+1}{2}\right)=\sum_{r=0}^{7}\left(\begin{array}{l}
14 \\
2 r
\end{array}\right) D_{2 r} x^{14-2 r},
$$

where

$$
D_{2 r}=\left(2-2^{2 r}\right) B_{2 r} .
$$

By the Staudt-Clausen theorem the denominator of $D_{2 r}$ is odd; moreover, if $p$ is an odd prime and $r>0$,

$$
p D_{2 r} \equiv\left\{\begin{array}{rll}
-1 & (\bmod p) & (p-1 \mid 2 r), \\
0 & (\bmod p) & (p-1 \nmid 2 r) .
\end{array}\right.
$$

We first take $p=3$. Then

$$
-3 P(x) \equiv \sum_{r=0}^{6}\left(\begin{array}{l}
14 \\
2 r
\end{array}\right) x^{2 r} \quad(\bmod 3) .
$$

It is easily verified that

$$
\left(\begin{array}{c}
14 \\
2
\end{array}\right) \equiv\left(\begin{array}{c}
14 \\
12
\end{array}\right) \equiv 1, \quad\left(\begin{array}{c}
14 \\
4
\end{array}\right) \equiv\left(\begin{array}{c}
14 \\
10
\end{array}\right) \equiv-1, \quad\left(\begin{array}{c}
14 \\
6
\end{array}\right) \equiv\left(\begin{array}{c}
14 \\
8
\end{array}\right) \equiv 0(\bmod 3),
$$

Received February 18, 1965. Supported in part by NSF grant GP-1593. 
so that

$$
-3 P(x) \equiv x^{12}-x^{10}-x^{4}+x^{2}+1 \quad(\bmod 3) .
$$

Here we have made use of the familiar result that if

$$
\begin{aligned}
n & =n_{0}+n_{1} p+n_{2} p^{2}+\cdots \quad\left(0 \leqq n_{j}<p\right), \\
r & =r_{0}+r_{1} p+r_{2} p^{2}+\cdots \quad\left(0 \leqq r_{j}<p\right),
\end{aligned}
$$

then

$$
\left(\begin{array}{l}
n \\
r
\end{array}\right) \equiv\left(\begin{array}{l}
n_{0} \\
r_{0}
\end{array}\right)\left(\begin{array}{l}
n_{1} \\
r_{1}
\end{array}\right)\left(\begin{array}{l}
n_{2} \\
r_{2}
\end{array}\right) \cdots \quad(\bmod p),
$$

where $p$ is a prime.

Next we verify that

$$
\begin{aligned}
& x^{12}-x^{10}-x^{4}+x^{2}+1 \equiv\left(x^{3}-x\right)^{4}+\left(x^{3}-x\right)^{2}+1 \\
& \equiv\left[\left(x^{3}-x\right)^{2}-1\right]^{2} \equiv\left(x^{3}-x-1\right)^{2}\left(x^{3}-x+1\right)^{2} \quad(\bmod 3) .
\end{aligned}
$$

The polynomials $x^{3}-x \pm 1$ are irreducible $(\bmod 3)$ since clearly neither has a linear factor. (The irreducibility is a special case of the irreducibility $(\bmod p$ ) of $x^{p}-x-k$, where $k$ is any integer not divisible by $p$.)

It is evident from (1) that

$$
P(x)=Q\left(x^{2}\right),
$$

where $Q(y)$ is a polynomial of degree 7 in $y$. Since

$$
\left(x^{3}-x-1\right)\left(x^{3}-x-1\right)=x^{2}\left(x^{2}-1\right)^{2}-1,
$$

it follows from (2) and (3) that

$$
-3 Q(y) \equiv\left(y(y-1)^{2}-1\right)^{2} \equiv\left(y^{3}-y^{2}-y-1\right)^{2} \quad(\bmod 3) .
$$

Also it is easily verified that $y^{3}+y^{2}+y-1$ is irreducible $(\bmod 3)$.

3 . We now take $p=13$. Then we find that

$$
P(x) \equiv x^{14}+\left(\begin{array}{c}
14 \\
2
\end{array}\right) D_{12} x^{2}+D_{14} \quad(\bmod 13) .
$$

By Kummer's congruence [3, Ch. 14]

$$
\frac{B_{14}}{14} \equiv \frac{B_{2}}{2} \equiv \frac{1}{12} \equiv-1 \quad(\bmod 13),
$$

so that

$$
D_{14}=\left(2-2^{14}\right) B_{14} \equiv 2 \quad(\bmod 13) .
$$

By the Staudt-Clausen theorem

$$
13 D_{12} \equiv-1 \quad(\bmod 13) .
$$

Thus (6) becomes

$$
P(x) \equiv x^{14}-\frac{1}{2} x^{2}+2 \quad(\bmod 13) .
$$


If $a$ is a rational integer, (7) gives

$$
P(a) \equiv \frac{1}{2} a^{2}+2 \equiv \frac{1}{2}(a+3)(a-3) \quad(\bmod 13) .
$$

Also, since

$$
P^{\prime}(x) \equiv x^{13}-x \quad(\bmod 13),
$$

it follows that 3 and -3 are zeros of $P(x)$ of multiplicity two.

In terms of $Q(y)$, as defined by (4), we have

$$
Q(y) \equiv(y+4)^{2} Q_{1}(y) \quad(\bmod 13)
$$

where $Q_{1}(y)$ is a polynomial of degree 5 in $y$ that has no linear factors. We shall now prove that $Q_{1}(y)$ has no quadratic factors $(\bmod 13)$. For assume that

$$
Q(a+\theta)=0 \quad\left(\theta^{2} \in \mathrm{GF}(13), \theta \notin \mathrm{GF}(13)\right),
$$

where $a$ is some rational integer and $\theta$ is a number of $\operatorname{GF}\left(13^{2}\right)$ that is not in $\operatorname{GF}(13)$. Then by (7)

so that

$$
Q(a+\theta)=(a+\theta)^{7}-\frac{1}{2}(a+\theta)+2=0,
$$

$$
(a+\theta)^{7}=\frac{1}{2} a-2+\frac{1}{2} \theta .
$$

Squaring both sides of this equation we get, since

$$
\begin{aligned}
(a+\theta)^{14} & =(a+\theta)\left(a+\theta^{13}\right)=(a+\theta)(a-\theta), \\
a^{2}-\theta^{2} & =\left(\frac{1}{2} a-2\right)^{2}+\frac{1}{2}(a-4) \theta+\frac{1}{4} \theta^{2} .
\end{aligned}
$$

This evidently implies $a=4, \theta^{2}=5$. The quadratic $y^{2}+5 y-2$ has the roots $4 \pm \sqrt{ } 5$ and is irreducible over $\mathrm{GF}(13)$. Thus if $(9)$ holds $Q(y)$ must be divisible by $y^{2}+5 y-2$; it is however readily verified that this is not the case.

It follows at once from the above discussion that $Q_{1}(y)$ is irreducible $(\bmod 13)$.

4. Returning to (1) we find that

$$
P(x) \equiv\left(x^{7}-x\right)^{2} \quad(\bmod 7) .
$$

By Kummer's congruence

$$
\frac{B_{14}}{14} \equiv \frac{B_{2}}{2} \equiv 3 \quad(\bmod 7),
$$

so that the numerator of $B_{14}$ is divisible by 7 but not by $7^{2}$. Since

$$
D_{14}=\left(2-2^{14}\right) B_{14},
$$

the same is true of $D_{14}$. Hence $P(x)$ is a polynomial with coefficients that are integral $(\bmod 7)$ and with constant term divisible by 7 but not by $7^{2}$; moreover $P(-x)=P(x)$. Now assume a factorization

$$
P(x)=P_{1}(x) P_{2}(x) \cdots P_{k}(x)
$$

where the $P_{j}(x)$ are normalized irreducibles with rational coefficients that are integral $(\bmod p)$. Exactly one of the $P_{j}(x)$, say $P_{1}(x)$, has constant term divisible by 7 . Replacing $x$ by $-x$ in (10) we infer that $P_{1}(-x)=P_{1}(x)$ and therefore 
$P_{1}(x)=Q_{1}\left(x^{2}\right)$. Hence the reducibility of $P(x)$ over the rational field implies the reducibility of $Q(x)$.

On the other hand, by (5) and (8),

$$
\left\{\begin{aligned}
-3 Q(y) & \equiv\left(y^{3}+y^{2}+y-1\right)^{2} \quad(\bmod 3), \\
Q(y) & \equiv(y+4)^{2} Q_{1}(y) \quad(\bmod 13),
\end{aligned}\right.
$$

where the factors on the right are irreducible for the respective moduli. Since $Q_{1}(y)$ is of degree 5 , it follows that $Q(y)$ is irreducible over the rationals. Therefore, by the preceding paragraph, $P(x)$ is also irreducible.

5. We remark that

$$
2 B_{14}(x) \equiv\left(x^{3}+x+1\right)^{2}\left(x^{3}+x^{2}+1\right)^{2} \quad(\bmod 2) ;
$$

the cubics $x^{3}+x+1, x^{3}+x^{2}+1$ are irreducible $(\bmod 2)$. We have also

$$
-5 B_{14}(x) \equiv\left(x^{5}-x\right)^{2} \quad(\bmod 5) .
$$

It can be verified that

$$
Q(y) \equiv y^{7}-3 y^{6}-3 y^{2}+4 y-4 \quad(\bmod 11)
$$

and that $Q(y)$ has no linear factors $(\bmod 11)$. However the complete factorization of $Q(y)(\bmod 11)$ has not been obtained.

We observe that for an arbitrary prime $p>3$, the polynomial

$$
P_{2 p}(x)=2^{2 p} B_{2 p}\left(\frac{x+1}{2}\right)=\sum_{r=0}^{p}\left(\begin{array}{l}
2 p \\
2 r
\end{array}\right) D_{2 r} x^{2 p-2 r}
$$

has coefficients integral $(\bmod p)$; indeed

$$
P_{2 p}(x) \equiv\left(x^{p}-x\right)^{2} \quad(\bmod p) .
$$

Moreover the constant term $D_{2 p}$ is divisible by $p$ and not by $p^{2}$. It follows, exactly as in the special case $p=7$, that to prove the irreducibility over the rationals of $P_{2 p}(x)$ it suffices to prove the irreducibility of $Q_{p}(y)$, where

$$
Q_{p}\left(x^{2}\right)=P_{2 p}(x) \text {. }
$$

Duke University,

Durham, North Carolina

1. L. Carlitz, "Note on irreducibility of the Bernoulli and Euler polynomials," Duke Math. J., v. 19, 1952, pp. 475-481. MR 14, 163.

2. P. J. McCARTHY, "Irreducibility of certain Bernoulli polynomials," Amer. Math. Monthly v. 68,1961 , pp. $352-353$. MR 23, * A1625.

3. N. NiElsen, Traité Elémentaire des Nombres de Bernoulli, Paris, 1923.

4. N. E. NöRLUNd, Vorlesungen über Differenzenrechnung, Berlin, 1924. 